\title{
ONE-DIMENSIONAL POLYNOMIAL MAPS, PERIODIC POINTS AND MULTIPLIERS
}

\author{
YURI G. ZARHIN (ZARKHIN)
}

\begin{abstract}
We discuss tangent maps related to the multipliers of periodic points of a typical one-dimensional polynomial map. UDC 517.535.2, 517.927.7
\end{abstract}

\section{Definitions, Notation, Statements}

We write $\mathbb{C}$ for the field of complex numbers. For every positive integer $m$ let us consider the affine space $\mathbf{A}^{m}=\mathbb{C}^{m}$ of all monic complex polynomials of degree $m$

$$
u(x)=x^{m}+\sum_{i=0}^{m-1} a_{i} x^{i}
$$

with coefficients $a=\left(a_{0}, \ldots, a_{m-1}\right) \in \mathbb{C}^{m}=\mathbf{A}^{m}$. It is convenient to identify the tangent space $\mathbb{C}^{m}$ to $u(x) \in \mathbf{A}^{m}$ with the space of all polynomials $p(x)$ of degree $\leq m-1$. Namely, to a polynomial $p(x)=\sum_{i=0}^{m-1} c_{i} x^{i}$ one assigns the tangent vector $\left(c_{0}, \ldots, c_{m-1}\right) \in \mathbb{C}^{m}$ that corresponds to "the tangency class at $u(x)$ of the curve" $\epsilon \rightarrow u(x)+\epsilon \cdot p(x) \in \mathbf{A}^{m}$ [8, Part II, Ch. III, Sect. 8, pp. 81-82].

Let $P_{m} \subset \mathbf{A}^{m}$ be the everywhere dense Zariski-open affine subset that consists of all polynomials without multiple roots. Let $f(x)=x^{m}+\sum_{i=0}^{m-1} a_{i} x^{i} \in P_{m}$ and let us choose a root $\alpha$ of $f(x)$. Locally (with respect to $a$ ), one may view $\alpha$ (using Implicit Function Theorem) as a holomorphic (univalued) function in $a=\left(a_{0}, \ldots, a_{m-1}\right)$. We have ([11, Sect. 2])

$$
d \alpha / d a_{i}=-\left[f^{\prime}(\alpha)\right]^{-1} \alpha^{i}
$$

(Since $\alpha$ is a simple root of $f(x)$, we have $f^{\prime}(\alpha) \neq 0$.) We also have (ibid)

$$
d f^{\prime}(\alpha) / d a_{i}=i \alpha^{i-1}-\left[f^{\prime}(\alpha)\right]^{-1} \alpha^{i} f^{\prime \prime}(\alpha)
$$

(of course, if $i=0$ then the first term disappears). Using these formulas, let us compute the differential $d \mathrm{~N}: \mathbb{C}^{m} \rightarrow \mathbb{C}($ at $f(x))$ of locally defined holomorphic function

$$
\mathrm{N}: P_{m} \rightarrow \mathbb{C}, f(x) \mapsto f^{\prime}(\alpha) .
$$

It follows that $d \mathrm{~N}$ sends the tangent vector $p(x)=\sum_{i=0}^{m-1} c_{i} x^{i}$ to the number

$$
d \mathrm{~N}(p(x))=\sum_{i=0}^{m-1} c_{i} \frac{d f^{\prime}}{d a_{i}}(\alpha)=p^{\prime}(\alpha)-\left[f^{\prime}(\alpha)\right]^{-1} p(\alpha) f^{\prime \prime}(\alpha) .
$$

This work was partially supported by a grant from the Simons Foundation (\#246625 to Yuri Zarkhin). 
Example 1.1. Suppose that $m \geq 3$ and $f(x)=x^{m}-x$. Then $\alpha$ is either zero or $(m-1)$ th root of unity. If $\alpha=0$ then $f^{\prime \prime}(0)=0$ and

$$
d \mathrm{~N}(p(x))=p^{\prime}(0)=c_{1} .
$$

The gradient of $\mathrm{N}$ at $f(x)=x^{m}-x$ (with respect to the root 0 ) is

$$
Q_{1}(0)=(0,1, \ldots, 0) \in \mathbb{C}^{m} \text {. }
$$

If $\alpha^{m-1}=1$ then

$$
\begin{gathered}
f^{\prime}(\alpha)=m \alpha^{m-1}-1=m-1, \\
f^{\prime \prime}(\alpha)=m(m-1) \alpha^{m-2}=m(m-1) / \alpha,
\end{gathered}
$$

and

$$
d \mathrm{~N}(p(x))=p^{\prime}(\alpha)-\frac{m p(\alpha)}{\alpha} .
$$

The gradient of $\mathrm{N}$ at $f(x)=x^{m}-x$ (with respect to the root $\alpha$ ) is

$$
Q_{1}(\alpha)=\left(-\frac{m}{\alpha},(1-m),(2-m) \alpha, \ldots,-\alpha^{m-2}\right) \in \mathbb{C}^{n} .
$$

Let $n \geq 2$ be an integer and $g(x) \in \mathbb{C}[x]$ a degree $n$ monic polynomial with complex coefficients. For every positive integer $r$ we denote by $g^{\circ r}(x)$ the composition $g(\ldots g(x))$ ( $r$ times). Clearly, $g^{\circ r}(x)$ is a degree $n^{r}$ monic polynomial with complex coefficients. Let us consider the polynomial map

$$
G: \mathbb{C} \rightarrow \mathbb{C}, z \mapsto g(z) .
$$

Clearly, the fixed points of $G$ are exactly the roots of $g(x)-x$ while the roots of $g^{\circ r}(x)-x$ are exactly the points of period (dividing) $r$.

Example 1.2. If $g(x)=x^{n}$ then $g^{\circ r}(x)=x^{n^{r}}, g^{\circ r}(x)-x=x^{n^{r}}-x$.

We write $Z_{n, r} \subset \mathbf{A}^{n}$ for the everywhere dense Zariski-open affine subset that consists of all monic degree $n$ polynomials $g(x)$ such that $g^{\circ r}(x)-x$ lies in $P_{n^{r}}$ (i.e., does not have multiple roots). For example, $x^{n} \in Z_{n, r}$ for all $r$. Clearly, for every positive integer $m$

$$
Z_{m, 1}=\left\{f(x)+x \mid f(x) \in P_{m}\right\} .
$$

It is also clear that the holomorphic map

$$
U_{m}: Z_{m, 1} \rightarrow P_{m}, h(x) \mapsto h(x)-x
$$

is a holomorphic isomorphism, whose tangent map

$$
d U_{n}: \mathbb{C}^{m} \rightarrow \mathbb{C}^{m}
$$

is the identity map at all points of $Z_{m, 1}$.

Let us consider a locally defined holomorphic function

$$
\mathrm{M}^{r}: Z_{n, r} \rightarrow Z_{n^{r}, 1} \rightarrow P_{n^{r}} \rightarrow \mathbb{C}, g(x) \mapsto g^{\circ r}(x) \stackrel{U_{n^{r}}}{\longmapsto} g^{\circ r}(x)-x \stackrel{\mathrm{N}}{\mapsto}\left[g^{\circ r}(x)-x\right]^{\prime}(\alpha)
$$

where $\alpha$ is a root of $g^{\circ r}(x)-x$. We are going to discuss its differential

$$
d \mathrm{M}_{\mid g(x)}^{r}: \mathbb{C}^{n} \rightarrow \mathbb{C},
$$

paying special attention to the computation of the corresponding gradient

$$
\operatorname{grad}\left(\mathrm{M}^{r}\right)_{\mid g(x)} \in \mathbb{C}^{n}
$$

at the point $g(x)=x^{n} \in Z_{n, r}$. In what follows we denote this gradient by $Q_{r}(\alpha)$. This notation is compatible with our previous notation for $Q_{1}(\alpha)$ in Example 1.1. 
Remark 1.3. Let us consider the locally defined multiplier function

$$
\text { Mult }^{r}: Z_{n, r} \rightarrow Z_{n^{r}, 1} \rightarrow \mathbb{C}, g(x) \longmapsto\left[g^{\circ r}\right]^{\prime}(\alpha) .
$$

Clearly, $\mathrm{M}^{r}(g)=\operatorname{Mult}^{r}(g)-1$. It follows that the differentials $d \mathrm{M}^{r}$ and $d \mathrm{Mult}^{r}$ everywhere coincide. In other words

$$
\operatorname{grad}\left(\mathrm{M}^{r}\right)_{\mid g(x)}=\operatorname{grad}\left(\operatorname{Mult}^{r}\right)_{\mid g(x)} \forall g(x) \in Z_{n, r} .
$$

1.4. In order to state our main results, first notice that if $g(x) \in \in Z_{n, r}$ and $\nu(n, r)$ is the number of of all orbits of length $r$ for the map $z \mapsto g(z)$ then

$$
\nu(n, r) \geq \frac{n}{r}
$$

(see Subsection 4.3). Second, let us consider a positive integer $\ell$ and a sequence $\left\{r_{1}, \ldots, r_{\ell}\right\}$ of $\ell$ positive integers. Let $Z\left(n, \ell ; r_{1}, \ldots, r_{\ell}\right)$ be the intersection of all $Z_{n, r_{i}}$; it is a nonempty Zariski-open affine subset in $\mathbf{A}^{n}$ that contains $g(x)=x^{n}$. Let $g(x) \in Z\left(n, \ell ; r_{1}, \ldots, r_{\ell}\right)$. For each $i$ pick a complex number $\beta_{i}$ that is a periodic point of $G: z \mapsto g(z)$ of exact period $r_{i}$. Locally (with respect to $g$ ), each $\beta_{i}$ is a holomorphic function (in the coefficients of $g(x)$.)

Suppose that $\beta_{1}, \ldots \beta_{\ell}$ belong to distinct orbits of $z \mapsto g(z)$. Let us consider the following $\ell$ locally defined holomorphic functions

$$
\operatorname{Mult}_{\beta_{i}, r_{i}}: Z\left(n, \ell ; r_{1}, \ldots, r_{\ell}\right) \rightarrow \mathbb{C}, g(x) \mapsto\left[g^{\circ r_{i}}\right]^{\prime}\left(\beta_{i}\right) .
$$

Let $Z^{0}\left(n, \ell ; r_{1}, \ldots, r_{\ell}\right)$ be the set of all polynomials $g(x) \in Z\left(n, \ell ; r_{1}, \ldots, r_{\ell}\right)$ such that the $\ell$-element set

$$
\left\{\operatorname{grad}\left(\operatorname{Mult}_{\beta_{i}, r_{i}}\right)_{\mid g(x)} \in \mathbb{C}^{n} \mid 1 \leq i \leq \ell\right\}
$$

of gradients of Mult $_{\beta_{i}, r_{i}}$ 's at $g(x)$ is linearly independent in $\mathbb{C}^{n}$ for every choice of $\left\{\beta_{1}, \ldots, \beta_{\ell}\right\}$. Clearly, $Z^{0}\left(n, \ell ; r_{1}, \ldots, r_{\ell}\right)$ is an open subset of $Z\left(n, \ell ; r_{1}, \ldots, r_{\ell}\right)$ and therefore of $\mathbf{A}^{n}$ in complex topology. However, this set may be empty; e.g., when $\ell \geq n$.

The following statements are main results of this paper.

Theorem 1.5. The set $Z^{0}\left(n, \ell ; r_{1}, \ldots, r_{\ell}\right)$ is a Zariski-open subset of $Z\left(n, \ell ; r_{1}, \ldots, r_{\ell}\right)$ and therefore of $\mathbf{A}^{n}$.

Theorem 1.6. Suppose that $n \geq 3$. Assume that $\sum_{i=1}^{\ell} r_{i} \leq n$. If $r_{j}=1$ for some $j$ then we assume additionally that $\sum_{i=1}^{\ell} r_{i}<n$.

Then $Z^{0}\left(n, \ell ; r_{1}, \ldots, r_{\ell}\right)$ contains $g(x)=x^{n}$ and therefore is nonempty.

Notice that under the notation and assumptions of Theorem 1.6. if $r$ is a positive integer and $l(r)$ is the number of $i$ 's with $r_{i}=r$ then

$$
l(r) \leq \frac{n}{r} \leq \nu(n, r) .
$$

Combining Theorems 1.5 and 1.6, we obtain the following statement.

Corollary 1.7. Suppose that $n \geq 3$. Assume that $\sum_{i=1}^{\ell} r_{i} \leq n$. If $r_{j}=1$ for some $j$ then we assume additionally that $\sum_{i=1}^{\ell} r_{i}<n$.

Then $Z^{0}\left(n, \ell ; r_{1}, \ldots, r_{\ell}\right)$ is a Zariski-open everywhere dense subset of $Z\left(n, \ell ; r_{1}, \ldots, r_{\ell}\right)$ that contains $g(x)=x^{n}$. 
Example 1.8. Suppose that $\ell=n-1$ and all $r_{i}=1$ (i.e., all the $\beta_{i}$ involved are fixed points). It follows from results of [11, 7] that

$$
Z^{0}(n, n-1 ; 1, \ldots, 1)=Z(n, n-1 ; 1, \ldots, 1)=Z_{n, 1} .
$$

Remark 1.9. In the notation and assumptions of Corollary 1.7, let us consider the locally defined holomorphic map

$$
Z\left(n, \ell ; r_{1}, \ldots, r_{\ell}\right) \rightarrow \mathbb{C}^{\ell}
$$

defined by the collection of functions $\left\{\operatorname{Mult}_{\beta_{i}, r_{i}}\right\}_{i=1}^{\ell}$. Corollary 1.7 asserts that this map has (maximal) rank $\ell$ on a nonempty Zariski-open subset

$$
Z^{0}\left(n, \ell ; r_{1}, \ldots, r_{\ell}\right) \subset Z\left(n, \ell ; r_{1}, \ldots, r_{\ell}\right)
$$

for every choice of periodic points $\left\{\beta_{1}, \ldots, \beta_{\ell}\right\}$. It would be interesting to study its image. For the case of fixed points (i.e., when all $r_{i}=1$ ), see [3].

Notice that Remark 1.9 gives a partial answer to a question of Yu.S. Ilyashenko, who was interested in the case of two orbits, in connection with [1, 2].

Remark 1.10. In the case of two orbits, it turns out (see Examples 2.3, 2.4 and Remark 2.5 below) that $g(x)=x^{n}$ does not belong to $Z\left(n, 2 ; r_{1}, r_{2}\right)$ if either $r_{1}=$ $r_{2}=n-1$ or $r_{1}=1, r_{2}=n-1$. It would be interesting to find out whether in these cases $Z\left(n, 2 ; r_{1}, r_{2}\right)$ is empty.

The paper is organized as follows. In Section 2 we compute explicitly the differentials $d \operatorname{Mult}_{\beta, r}$ at $g(x)=x^{n}$ where $\beta$ is a $\left(n^{r}-1\right)$ th root of unity. This allows us to write down explicitly the corresponding gradients $Q_{r}(\beta) \in \mathbb{C}^{n}$. Now Theorem 1.6 becomes equivalent to an assertion that the corresponding set of vectors $\left\{Q_{r_{i}}\left(\beta_{i}\right)\right\}$ (and $Q_{1}(0)$ if one of $r_{i}$ is 1 ) is linearly independent in $\mathbb{C}^{n}$. We prove this assertion in Section 3, Using standard properties of finite maps (9, Ch. 1], [5, Sect. 8]), we prove Theorem 1.5 in Section 4.

\section{Computations of tangent maps}

Lemma 2.1. Let us consider the holomorphic map $\Phi_{n, r}: \mathbf{A}^{n} \rightarrow \mathbf{A}^{n^{r}}$ that sends a degree $n$ monic polynomial $g(x)$ to the monic degree $n^{r}$ polynomial $g^{\text {or }}(x)$. Then the tangent map $d \Phi_{n, r}$ at $g(x)=x^{n}$ is as follows. It sends a tangent vector $x^{k}$ (at the point $x^{n}$ ) to the tangent vector

$$
p_{r, k}(x):=\sum_{i=1}^{r} n^{r-i} x^{n^{r}-n^{i}+n^{i-1} k}
$$

(at the point $x^{n^{r}}$ ). In particular,

$$
\begin{gathered}
p_{r, 0}(x):=\sum_{i=1}^{r} n^{r-i} x^{n^{r}-n^{i}}=n^{r-1} x^{n^{r}-n}+n^{r-2} x^{n^{r}-n^{2}}+\ldots, \\
p_{r, 1}(x):=\sum_{i=1}^{r} n^{r-i} x^{n^{r}-n^{i}+n^{i-1}}=n^{r-1} x^{n^{r}-n+1}+n^{r-2} x^{n^{r}-n^{2}+n}+\ldots
\end{gathered}
$$

and

$$
\operatorname{deg}\left(p_{r, 0}\right)=n^{r}-n, \operatorname{deg}\left(p_{r, 1}\right)=n^{r}-n+1, \operatorname{deg}\left(p_{r, k}\right)=n^{r}-n+k .
$$


Proof. Notice that $p_{1, k}(x)=x^{k}$ and for all positive integers $r$

$$
p_{r+1, k}(x)=n x^{(n-1) n^{r}} p_{r, k}(x)+x^{k n^{r}} .
$$

Induction by $r$. We need to prove that if $g^{[\epsilon]}(x)=x^{n}+\epsilon x^{k}$ then $\left[g^{[\epsilon]}\right]^{\circ r}(x)=$ $x^{n^{k}}+\epsilon p_{r, k}(x)+O\left(\epsilon^{2}\right)$. If $r=1$ then it is obvious. Assume that this assertion is true for $r$ and let us check it for $r+1$. We have

$$
\begin{gathered}
{\left[g^{[\epsilon]}\right]^{\circ(r+1)}(x)=} \\
\left\{\left[g^{[\epsilon]}\right]^{\circ r}(x)\right\}^{n}+\epsilon\left\{\left[g^{[\epsilon]}\right]^{\circ r}(x)\right\}^{k}=\left(x^{n^{r}}+\epsilon p_{r, k}(x)+O\left(\epsilon^{2}\right)\right)^{n}+\epsilon\left(x^{n^{r}}+\epsilon p_{r, k}(x)+O\left(\epsilon^{2}\right)\right)^{k}= \\
x^{n^{r+1}}+\epsilon n x^{(n-1) n^{r}} p_{r, k}(x)+\epsilon x^{k n^{r}}+O\left(\epsilon^{2}\right)= \\
x^{n^{r+1}}+\epsilon\left\{n x^{(n-1) n^{r}} p_{r, k}(x)+x^{k n^{r}}\right\}+O\left(\epsilon^{2}\right)=x^{n^{r+1}}+\epsilon p_{r, k+1}(x)+O\left(\epsilon^{2}\right) .
\end{gathered}
$$

Since all $p_{r, k}$ (for given $n$ and $r$ ) have distinct degrees, the set $\left\{p_{r, 0}, \ldots, p_{r, n-1}\right\}$ is linearly independent. This means that the rank of the tangent map to $\Phi_{n, r}$ at $g(x)=x^{n}$ is $n$, i.e. the tangent map at this point is injective and its image coincides with

$$
\oplus_{k=0}^{n-1} \mathbb{C} \cdot p_{r, k}
$$

2.2. Suppose that $n^{r} \geq 3$. Let us compute the differential

$$
d \mathrm{Mult}^{r}=d \mathrm{M}^{r}=d\left(\mathrm{M}_{n, r}\right)=d \mathrm{M} \circ d \Phi_{n, r}
$$

at $g(x)=x^{n} \in Z_{n, r}$. Clearly,

$$
\Phi_{n, r}\left(x^{n}\right)=x^{n^{r}} \in P_{m}
$$

with $m=n^{r}$. Let $\alpha$ be a nonzero root of $x^{m}-x$, i.e., $\alpha^{n^{r}-1}=1$. Using Lemma 2.1 and Example 1.1, we obtain the following. The image

$$
q_{r, k}(\alpha):=d \operatorname{Mult}_{\mid g(x)=x^{n}}^{r}\left(x^{k}\right)
$$

of tangent vector $x^{k}$ to $g(x)=x^{n} \in Z_{n, r}$ is

$$
\begin{gathered}
p_{r, k}^{\prime}(\alpha)-\frac{n^{r} p_{r, k}(\alpha)}{\alpha}= \\
\alpha^{-1} \sum_{i=1}^{r}\left(n^{r}-n^{i}+n^{i-1} k\right) n^{r-i} \alpha^{n^{r}-n^{i}+n^{i-1} k}-\alpha^{-1} \sum_{i=1}^{r} n^{r} n^{r-i} \alpha^{n^{r}-n^{i}+n^{i-1} k}= \\
\alpha^{n^{r}-1} \sum_{i=1}^{r}\left[n^{2 r-i}-n^{r}+k n^{r-1}-n^{2 r-i}\right] \alpha^{-n^{i}+n^{i-1} k}=-\left(n^{r}-k n^{r-1}\right) \sum_{i=1}^{r} \alpha^{-n^{i}+n^{i-1} k}= \\
-(n-k) n^{r-1} \sum_{i=1}^{r} \alpha^{-n^{i}+n^{i-1} k}=-(n-k) n^{r-1} \sum_{i=1}^{r}\left(\frac{1}{\alpha^{n^{i-1}}}\right)^{n-k} .
\end{gathered}
$$

In other words,

$$
q_{r, k}(\alpha)=d \operatorname{Mult}_{\mid g(x)=x^{n}}^{r}\left(x^{k}\right)=-(n-k) n^{r-1} \sum_{i=1}^{r}\left(1 / \alpha^{n^{i-1}}\right)^{n-k} .
$$

Notice that

$$
q_{r, k}(\alpha)=q_{r, k}\left(\alpha^{n}\right), q_{r, 0}(\alpha)=n \cdot q_{r, n-1}(\alpha) .
$$

It follows that the gradient of Mult ${ }^{r}$ at $g(x)=x^{n}$ (with respect to $\alpha$ ) is $Q_{r}(\alpha)=\left(q_{r, 0}(\alpha), q_{r, 1}(\alpha), \ldots, q_{r, n-1}(\alpha)\right)=\left(n q_{r, n-1}(\alpha), q_{r, 1}(\alpha), \ldots, q_{r, n-1}(\alpha)\right) \in \mathbb{C}^{n}$. 
Clearly,

$$
Q_{r}(\alpha)=Q_{r}\left(\alpha^{n}\right)=\cdots=Q_{r}\left(\alpha^{n^{r-1}}\right) .
$$

Let $\mathcal{O}(\alpha)=\left\{\alpha, \alpha^{n}, \ldots, \alpha^{n^{r-1}}\right\}$ be the orbit of $\alpha$ with respect to $z \mapsto z^{n}$ and let $d(\alpha)$ be the cardinality of the set $\mathcal{O}(\alpha)$. Clearly, $d(\alpha)$ is a positive integer that divides $r$ and

It is also clear that

$$
\beta^{d(\alpha)}=\beta \forall \beta \in \mathcal{O}(\alpha) .
$$

$$
q_{r, k}(\alpha)=-\frac{r}{d(\alpha)}(n-k) n^{r-1} \sum_{\beta \in \mathcal{O}(\alpha)}(1 / \beta)^{n-k} .
$$

This implies that

$$
Q_{r}(\alpha)=\frac{r n^{r}}{d(\alpha) n^{d(\alpha)}} \cdot Q_{d(\alpha)}(\alpha)
$$

Example 2.3. Suppose that $n=3$ and $r=2$. Then $n^{r}-1=8$. Let $\alpha$ be a 8th root of unity that is not \pm 1 . Then $\alpha$ is a periodic point of exact period 2 for $z \mapsto z^{3}$. We have

$$
Q_{2}(\alpha)=-3^{1} \cdot\left(3\left[\frac{1}{\alpha}+\frac{1}{\alpha^{3}}\right], 2\left[\frac{1}{\alpha^{2}}+\frac{1}{\alpha^{6}}\right],\left[\frac{1}{\alpha}+\frac{1}{\alpha^{3}}\right]\right) .
$$

So, if $\alpha$ is a primitive fourth root of unity, i.e.,

$$
\alpha^{2}=-1, \alpha= \pm \mathbf{i}
$$

then

and

$$
\frac{1}{\alpha}+\frac{1}{\alpha^{3}}=0, \frac{1}{\alpha^{2}}+\frac{1}{\alpha^{6}}=-2
$$

$$
Q_{2}(\alpha)=-3 \cdot(0,-2,0)=(0,6,0) .
$$

(Notice that $\mathbf{i}$ and $-\mathbf{i}$ lie in the same orbit.)

If $\alpha$ is a primitive 8 th root of unity then

and therefore

$$
\alpha^{4}=-1,1+\frac{1}{\alpha^{4}}=0
$$

$\left.Q_{2}(\alpha)=-3 \cdot\left(3\left[\frac{1}{\alpha}+\frac{1}{\alpha^{3}}\right], 0,\left[\frac{1}{\alpha}+\frac{1}{\alpha^{3}}\right]\right)=-\frac{3}{\alpha^{3}} \cdot\left(3\left[\alpha^{2}+1\right], 0, \alpha^{2}+1\right)\right)=-\frac{3\left(\alpha^{2}+1\right)}{\alpha^{3}} \cdot(3,0,1)$.

Now if we put $\beta=\alpha^{-1}$ then $\alpha$ and $\beta$ are primitive 8 th roots of unity that do not belong to the same orbit while $Q_{2}(\alpha)$ and $Q_{2}(\beta)$ generate the same line $\mathbb{C} \cdot(3,0,1)$ in $\mathbb{C}^{3}$. This implies that $Z(3,2 ; 2,2)$ does not contain $g(x)=x^{3}$.

Example 2.4. Suppose that $n=r+1$ and $r \geq 2$. Then for all positive integers $i$

$$
n^{i}=(1+r)^{i}=1+i \cdot r^{1}+\cdots+\left(\begin{array}{c}
i \\
j
\end{array}\right) r^{j}+\cdots+r^{i}
$$

It follows that $n^{i}$ is congruent to $1+i r$ modulo $r^{2}$. In particular, $n^{r}-1$ is divisible by $r^{2}=(n-1)^{2}$. It also follows that $\left(n^{i}-n\right) /(n-1)=n^{i-1}$ is congruent to $i-1$ modulo $r$ and therefore

$$
n^{i}-n \equiv(i-1) r \bmod r^{2} .
$$

Suppose that $\alpha$ is a primitive $r^{2}$ th root of unity. Then $\alpha^{n^{r}-1}=1$ and therefore

$$
\alpha^{n^{r}}=\alpha,
$$


i.e., $\alpha$ is a periodic point for the map $z \mapsto z^{n}$. Clearly, its period divides $r$. On the other hand, for all positive integers $i<r$ the power $n^{i}$ is not congruent to 1 modulo $r^{2}$ and therefore $\alpha^{n^{i}} \neq \alpha$. It follows that $\alpha$ has exact period $r$.

The number $\gamma:=\alpha^{1-n}=\alpha^{-r}$ is a primitive $r$ th root of unity. For each integer $k$ with $0 \leq k \leq n-1$ the number $\delta=\gamma^{n-k}$ is an $r$ th root of unity. Clearly, $\delta \neq 1$ if and only if $n-k \neq n-1$, i.e. $k \neq 1$. In particular, if $k \neq 1$ then $\sum_{i=1}^{r} \delta^{i}=0$.

We have

$$
\begin{gathered}
q_{r, k}(\alpha)=-(n-k) n^{r-1} \sum_{i=1}^{r}\left(\alpha^{-n^{i}}\right)^{n-k}= \\
-(n-k) n^{r-1} \cdot \alpha^{n(k-n)} \sum_{i=1}^{r}\left(\alpha^{n-n^{i}}\right)^{n-k}=-(n-k) n^{r-1} \alpha^{n(k-n)} \sum_{i=1}^{r} \delta^{i-1}= \\
-(n-k) n^{r-1} \delta^{-1} \alpha^{n(k-n)} \sum_{i=1}^{r} \delta^{i}=0
\end{gathered}
$$

if $k \neq 1$. On the other hand, if $k=1$ then $\delta=1$ and $q_{r, 1}(\alpha)=-r(n-1) n^{r-1} \gamma$. It follows that

$$
Q_{r}(\alpha)=-\left(0, r(n-1) n^{r-1} \gamma, 0, \ldots, 0\right)=-r(n-1) n^{r-1} \gamma \cdot Q_{1}(0) \in \mathbb{C}^{n} .
$$

This implies that $Z^{0}(r+1,2 ; r, 1)$ does not contain $g(x)=x^{n}$.

Remark 2.5. Suppose that $r>2$ and $n=r+1$. Example 2.4 tells us that if $\alpha$ and $\beta$ are primitive $r^{2}$ th roots of unity then $Q_{r}(\alpha)$ and $Q_{r}(\beta)$ generate the same line $\mathbb{C} \cdot(0,1, \ldots, 0)$ in $\mathbb{C}^{n}$. Since $r>2$, the number $\varphi\left(r^{2}\right)$ of primitive $r^{2}$ th roots of unity is strictly greater than $r$. (Here $\varphi$ is the Euler function.) In particular, we may choose such $\alpha$ and $\beta$ from different orbits (of length $r$ ) of the map $z \mapsto z^{n}$. It follows that $Z^{0}(r+1,2 ; r, r)$ does not contain $g(x)=x^{n}$.

\section{LINEAR INDEPENDENCE}

As was already pointed out, Theorem 1.6 is an immediate corollary of the following statement.

Theorem 3.1. Let $\ell$ be a positive integer. Let $\left\{r_{1}, \ldots r_{\ell}\right\}$ be a sequence of $\ell$ positive integers. Let $\left\{\alpha_{1}, \ldots, \alpha_{\ell}\right\}$ be a sequence of distinct complex numbers such that

$$
\alpha_{i}^{n^{r_{i}}-1}=1 \forall i=1, \ldots, \ell .
$$

Assume that $\left\{\alpha_{1}, \ldots, \alpha_{\ell}\right\}$ belong to different orbits of the map $z \mapsto z^{n}$. Then:

(i) the set of $\ell$ vectors $\left\{Q_{r_{1}}\left(\alpha_{1}\right), \ldots, Q_{r_{\ell}}\left(\alpha_{\ell}\right)\right\}$ in $\mathbb{C}^{n}$ is linearly independent if $n \geq \sum_{i=1}^{\ell} d\left(\alpha_{i}\right)$. In particular, if $\sum_{i=1}^{\ell} r_{i} \leq n$ then the $\ell$-tuple $\left\{Q_{r_{1}}\left(\alpha_{1}\right), \ldots, Q_{r_{\ell}}\left(\alpha_{\ell}\right)\right\}$ is linearly independent in $\mathbb{C}^{n}$.

(ii) If $n \geq 2+\sum_{i=1}^{\ell} d\left(\alpha_{i}\right)$ then the $(\ell+1)$-tuple $\left\{Q_{1}(0) ; Q_{r_{1}}\left(\alpha_{1}\right), \ldots, Q_{r_{\ell}}\left(\alpha_{\ell}\right)\right\}$ is a linearly independent set in $\mathbb{C}^{n}$. In particular, if $\sum_{i=1}^{\ell} r_{i}<n-1$ then the $(\ell+1)$-tuple $\left\{Q_{1}(0) ; Q_{r_{1}}\left(\alpha_{1}\right), \ldots, Q_{r_{\ell}}\left(\alpha_{\ell}\right)\right\}$ is a linearly independent set in $\mathbb{C}^{n}$.

Proof of Theorem 3.1. In the course of the proof we will use the following elementary statement that will be proven at the end of this section. 
Lemma 3.2. Let $d$ be a positive integer, $S$ a set of $d$ nonzero complex numbers. Let $c: S \rightarrow \mathbb{C}$ be a function such that for all positive integers $u=1, \ldots, d$

$$
\sum_{\beta \in S} \frac{c(\beta)}{\beta^{u}}=0 .
$$

Then $c(\beta)=0$ for all $\beta \in S$.

Let us continue to prove Theorem 3.1. Replacing each $r_{i}$ by $d\left(\alpha_{i}\right)$ we may and will assume that $r_{i}=d\left(\alpha_{i}\right)$, i.e., the orbit $\mathcal{O}\left(\alpha_{i}\right)$ of $\alpha_{i}$ consists of $r_{i}$ distinct elements (for all $i$ ). We also assume that $n \geq \sum_{i=1}^{\ell} r_{i}$.

Let $\left\{c_{1}, \ldots c_{\ell}\right\}$ be a sequence of $\ell$ complex numbers such that

$$
\sum_{i=1}^{\ell} c_{i} Q_{r_{i}}\left(\alpha_{i}\right)=0
$$

Let $S \subset \mathbb{C}$ be the (disjoint) union of all $\mathcal{O}\left(\alpha_{i}\right)$, which consists of $\left(\sum_{i=1}^{\ell} r_{i}\right)$ elements. Let us define a complex valued function $c$ on $S$ that assigns to $\alpha \in \mathcal{O}\left(\alpha_{i}\right)$ the complex number

$$
c(\alpha):=n^{r_{i}-1} c_{i}
$$

Then we obtain for all $k=0,1, \ldots, n-1$

$$
0=\sum_{i=1}^{\ell} c_{i} q_{r_{i}, k}\left(\alpha_{i}\right)=-(n-k) \sum_{\alpha \in S} c(\alpha)(1 / \alpha)^{n-k} .
$$

This implies that

$$
\sum_{\alpha \in S} c(\alpha)(1 / \alpha)^{u}=0
$$

for all positive integers $u=1, \ldots, n$.

It follows from Lemma 3.2 applied to $d=\sum_{i=1}^{\ell} r_{i}$ that all $c(\alpha)=0$. Since all $n^{r_{i}-1} \neq 0$, we conclude that all $c_{i}=0$. This proves (i).

Now assume that $n \geq 2+\sum_{i=1}^{\ell} r_{i}$. We are going to prove (ii). Let $\left\{c_{0}, c_{1}, \ldots c_{\ell}\right\}$ be a sequence of $(\ell+1)$ complex numbers such that

$$
c_{0} Q_{1}(0)+\sum_{i=1}^{\ell} c_{i} Q_{r_{i}}\left(\alpha_{i}\right)=0 \text {. }
$$

We have

$$
-c_{0} Q_{1}(0)=\sum_{i=1}^{\ell} c_{i} Q_{r_{i}}\left(\alpha_{i}\right) .
$$

Recall that all the coordinates of $Q_{1}(0)$ except the second one do vanish. This implies that

$$
0=\sum_{i=1}^{\ell} c_{i} q_{r_{i}, k}\left(\alpha_{i}\right)=-(n-k) \sum_{\alpha \in S} c(\alpha)(1 / \alpha)^{n-k}
$$

for all $k=0, \ldots n-1$ except $k=1$. It follows that

$$
\sum_{\alpha \in S} c(\alpha)(1 / \alpha)^{u}=0
$$


for all positive integers integers $u=1, \ldots, n-2$. Since $n-2 \geq d$, the same arguments with Lemma 3.2 as above prove that $c_{i}=0$ for all positive integers $i$ and therefore $-c_{0} Q_{1}(0)=0$, i.e., $c_{0}=0$.

Proof of Lemma 3.2. This Lemma is a variant of well-known classical results (e.g., see [10]). Let us consider the rational function

$$
X(t)=\sum_{\beta \in S} \frac{c(\beta)}{\beta-t}=\sum_{\beta \in S} \frac{c(\beta) / \beta}{1-\frac{t}{\beta}} .
$$

Clearly,

$$
X(t)=\frac{Q(t)}{\prod_{\beta \in S}(\beta-t)}
$$

where $Q(t)$ is a polynomial, whose degree does not exceed $d-1$. (Recall that $d=\#(S)$.) For each positive integer $u$, the number $\sum_{\beta \in S} c(\beta) / \beta^{u}$ is the $(u-1)$ th coefficient of the Taylor power series of $X(t)$ at the origin (see [4, Ch. 1, Sect. 2]). It follows that $X(t)$ has a zero of order $\geq d$ at the origin. This implies that $Q(t)$ is divisible by $t^{d}$ and therefore $Q(t)=0$, i.e. $X(t)=0$. Since $-c(\beta)$ is the residue of $X(t)$ at $t=\beta$ for all $\beta \in S$, we conclude that $c(\beta)=0$.

Remark 3.3. One may give even more elementary proof of Lemma 3.2, using the nondegeneracy of the Vandermonde matrix of size $d \times d$ for distinct numbers $\{1 / \beta \mid \beta \in S\}$.

\section{Openness in Zariski topology}

The aim of this Section is to prove Theorem 1.5. We will need the following well known easy statement.

Lemma 4.1. Let $n \geq 2$ be an integer and $g(x) \in \mathbb{C}[x]$ is a monic degree $n$ polynomial. Suppose that $g(x)-x$ has a multiple root say, $\alpha$. Then for all positive integers $r$ the complex number $\alpha$ is a multiple root of $g^{\circ r}(x)-x$.

Proof. We have

$$
g(\alpha)=\alpha, g^{\prime}(\alpha)=1
$$

It follows easily that

$$
g^{\circ r}(\alpha)=\alpha,\left[g^{\circ r}\right]^{\prime}(\alpha)=1
$$

This means that

$$
\left[g^{\circ r}(x)-x\right](\alpha)=0,\left[g^{\circ r}(x)-x\right]^{\prime}(\alpha)=0 .
$$

In other words, $\alpha$ is a multiple root of $g^{\circ r}(x)-x$.

Corollary 4.2. Let $m$ be a positive integer that divides $r$. Suppose that $g^{\circ m}(x)-x$ has a multiple root say, $\alpha$. Then $\alpha$ is a multiple root of $g^{\circ r}(x)-x$.

4.3. Let $g(x) \in Z_{n, r}$. If $m$ is a positive divisor of $r$ then Corollary 4.2 implies that $g(x) \in Z_{n, m}$. The number of periodic points of exact period $m$ for $z \mapsto g(z)$ is

$$
\nu_{n}(m)=\sum_{m^{\prime} \mid m} \mu\left(\frac{m}{m^{\prime}}\right) n^{m^{\prime}}
$$


where $\mu$ is the Möbius function [6, pp. 74-75]. In particular, the number of orbits of length $m$ equals

$$
d(n, m)=\frac{\nu_{n}(m)}{m}
$$

and therefore $\nu_{n}(m)$ is divisible by $m$. The explicit formula for $\nu_{n}(m)$ implies that $\nu_{n}(m)$ is also divisible by $n$ (ibid). It follows that $\nu_{n}(m)$ is divisible by $n m /(n, m)$ where $(n, m)$ is the greatest common divisor of $n$ and $m$. On the other hand, the number $\nu_{n}(m)$ is always positive. Indeed, $\nu_{n}(1)=n$ and for $1<m \leq 5$ we have $\nu_{n}(m)=n^{m}-n^{m / p}>0$ where $p$ is the only prime divisor of $m$. Now assume that $m>5$, i.e, $m \geq 6$. Notice that the points of exact period $m$ are exactly the roots of $g^{\circ m}(x)-x$ that are not roots of $g^{\circ(m / p)}(x)-x$ for any prime divisor $p$ of $m$. Since the number of prime divisors of $m$ does not exceed $\log _{2}(m)$,

$$
\nu_{n}(m) \geq n^{m}-n^{m / 2} \log _{2}(m) .
$$

It is easy to check that (under our assumptions on $n$ and $m$ ) we have $n^{m / 2} \geq$ $2^{m / 2}>\log _{2}(m)$ and therefore $\nu_{n}(m)$ is positive.

Since $\nu_{n}(m)$ is divisible by $\frac{n m}{(n, m)}$,

$$
\nu_{n}(m) \geq \frac{n m}{(n, m)}, d(n, m) \geq \frac{n}{(n, m)} \geq \frac{n}{m} .
$$

4.4. For each positive divisor $m$ of $r$ we pick a $d(n, m)$-element set $S_{m}$ and consider the corresponding $d(n, m)$-dimensional coordinate space $\mathbb{C}^{S_{m}}$ of all $\mathbb{C}$-valued functions on $S_{m}$.

Let us consider the Zariski-closed subset

$$
\hat{Z}_{n, r} \subset Z_{n, r} \times \prod_{m \mid r} \mathbb{C}^{S_{m}}
$$

that is cut out by the following equations imposed on

$$
\left\{g ; \phi_{m}: S_{m} \rightarrow \mathbb{C}, m \mid r\right\} \in Z_{n, r} \times \prod_{m \mid r} \mathbb{C}^{S_{m}} .
$$

For each $s \in S_{m}$ the complex number $\phi_{m}(s)$ is a periodic point, whose period divides $m$, with respect to $z \mapsto g(z)$, i.e. $g^{\circ m}\left(\phi_{m}(s)\right)=\phi_{m}(s)$. In addition, we require that

$$
g^{\circ r}(x)-x=\prod_{m \mid r}\left(\prod_{s \in S_{m}}\left(x-\phi_{m}(s)\right) \prod_{i=1}^{m-1}\left(x-g^{\circ i}\left(\phi_{m}(s)\right)\right)\right) .
$$

In other words, the coefficients of both polynomials in $x$ do coincide. Notice that

$$
g^{\circ r}\left(\phi_{m}(s)\right)-\phi_{m}(s)=0
$$

on $\hat{Z}_{n, r}$. In particular, all the coordinate functions $\phi_{m}(s)$ on $\hat{Z}_{n, r}$ are integral over the polynomial ring $\mathbb{C}\left[\mathbf{A}^{n}\right]=\mathbb{C}\left[a_{0}, \ldots, a_{n-1}\right]$, which is generated by the coefficients of $g(x)=x^{n}+\sum_{i=0}^{n-1} a_{i} x^{i}$.

Recall that $g^{\circ r}(x)-x$ has no multiple roots. It follows that each map $\phi_{m}$ : $S_{m} \rightarrow \mathbb{C}$ is injective, its image consists of elements of exact period $m$ while distinct elements of $S_{m}$ go under $\phi_{m}$ to distinct orbits of length $m$; in addition, every orbit of length $m$ contains exactly one element of $\phi_{m}\left(S_{m}\right)$. On the other hand, for any choice of an element $\zeta$ in every orbit of length $m$ (for each divisor $m$ of $r$ ) there is 
(exactly one) point of $\hat{Z}_{n, r}$ that lies "above" $g(x)$ and such that the corresponding $\phi_{m}\left(S_{m}\right)$ consists of these $\zeta$.

By construction, the projection map of affine varieties $\hat{Z}_{n, r} \rightarrow Z_{n, r}$ is surjective. In addition, this map is finite, because the ring of regular functions $\mathbb{C}\left[\hat{Z}_{n, r}\right]$ on $\hat{Z}_{n, r}$ is generated over $\mathbb{C}\left[Z_{n, r}\right] \supset \mathbb{C}\left[\mathbf{A}^{n}\right]=\mathbb{C}\left[a_{0}, \ldots, a_{n-1}\right]$ by the coordinate functions $\phi_{m}(s)$ that are integral over $\mathbb{C}\left[\mathbf{A}^{n}\right]$.

Now one may "lift" $\operatorname{Mult}^{r}=$ Mult $_{r, \beta}$ to globally defined functions on $\hat{Z}_{n, r}$. Namely, for each $s \in S_{r}$ the function

$$
\overline{\operatorname{Mult}}_{r, s}: \hat{Z}_{n, r} \rightarrow \mathbb{C},\left\{g ; \phi_{m}: S_{m} \rightarrow \mathbb{C}, m \mid r\right\} \mapsto \operatorname{Mult}_{r, \phi_{r}(s)}(g)
$$

is a globally defined regular function. If $\phi_{m}(s)$ and $\beta$ lie in the same orbit of length $m$ then this function coincides with the composition of projection map $\hat{Z}_{n, r} \rightarrow Z_{n, r}$ and Mult ${ }_{r, \beta}$. It is also clear that the vector function

$$
\overline{\operatorname{grad}}\left(\operatorname{Mult}_{r, s}\right): \hat{Z}_{n, r} \rightarrow \mathbb{C}^{n},\left\{g ; \phi_{m}: S_{m} \rightarrow \mathbb{C}^{n}, m \mid r\right\} \mapsto \operatorname{grad}\left(\operatorname{Mult}_{r, \phi_{r}(s)}\right)_{\mid g(x)}
$$

is a regular map that coincides with the composition of projection map $\hat{Z}_{n, r} \rightarrow Z_{n, r}$ and $\operatorname{grad}\left(\operatorname{Mult}_{r, \beta}\right): Z_{n, r} \rightarrow \mathbb{C}^{n}$ with $\beta=\phi_{r}(s)$.

Let $\ell$ be a positive integer that does not exceed $d(n, r)$. If $D$ is an $\ell$-element subset of $S_{r}$ let us consider the the subset $X_{D}$ of points $v \in \hat{Z}_{n, r}$ such that the collection of $\ell$ vectors $\left\{\overline{\operatorname{grad}}\left(\operatorname{Mult}_{r, s}\right)(v) \mid s \in D\right\}$ is linearly dependent in $\mathbb{C}^{n}$. Clearly, $X_{D}$ is a Zariski-closed subset in $\hat{Z}_{n, r}$. It follows that the union $X$ of all $X_{D}$ (where $D$ runs through all $\ell$-element subsets of $S_{r}$ ) is also closed in $\hat{Z}_{n, r}$. The finiteness of the projection map implies that the image $\bar{X}$ of $X$ in $Z_{n, r}$ is also Zariski-closed ([9, Ch. 1, Sect. 5.3]). On the other hand, one may easily check that $\bar{X}$ is the complement of $Z^{0}(n, \ell ; r, r, \ldots, r)$ in $Z(n, \ell ; r, r \ldots, r)=Z_{n, r}$. It follows that $Z^{0}(n, \ell ; r, r \ldots, r)$ is Zariski-open in $Z_{n, r}$. This proves Theorem 1.5 in the case when $r_{1}=r_{2}=\cdots=r_{\ell}$.

4.5. Now let us consider the general case. Let $d$ be the number of distinct elements in the sequence $\left\{r_{1}, \ldots, r_{\ell}\right\}$ and $R$ the corresponding $d$-element set of positive integers. For each $r \in R$ we denote by $l(r)$ the number of $i$ with $r_{i}=r$. If there is $r$ with $l(r)>d(n, r)$ then $Z\left(n, \ell ; r_{1}, r_{2}, \ldots, r_{\ell}\right)$ is empty. So further we assume that $l(r) \leq d(n, r)$ for all $r \in R$.

We write $\hat{Z}_{n, r}^{\prime}$ for the preimage of $Z\left(n, \ell ; r_{1}, r_{2}, \ldots, r_{\ell}\right)$ in $\hat{Z}_{n, r}$. The natural regular map

$$
\hat{Z}_{n, r}^{\prime} \rightarrow Z\left(n, \ell ; r_{1}, r_{2}, \ldots, r_{\ell}\right)
$$

is finite, because the base change of a finite map is finite [5, Sect. 8, Prop. 8.22]. Let us consider the fiber product $\hat{Z}_{n, R}$ of all $\hat{Z}_{n, r}^{\prime}$ (where $r$ runs through $R$ ) over $Z\left(n, \ell ; r_{1}, r_{2}, \ldots, r_{\ell}\right)$. If we write $\pi$ for the natural regular map

$$
\hat{Z}_{n, R} \rightarrow Z\left(n, \ell ; r_{1}, r_{2}, \ldots, r_{\ell}\right)
$$

then $\pi$ is finite, because the composition of finite maps is also finite [5, Sect. 8, Prop. 8.4 and 8.22]. We denote by $\pi_{r}$ the natural finite regular map

$$
\pi_{r}: \hat{Z}_{n, R} \rightarrow \hat{Z}_{n, r}^{\prime} \text {. }
$$

Now for each $r \in R$ pick a $l(r)$-element subset $D_{r}$ in $S_{r}$ and consider the subset $X_{\left\{D_{r} \mid r \in R\right\}}$ of points $v \in \hat{Z}_{n, R}$ such that the collection of $\left(\sum_{r \in R} l(r)\right)$ vectors

$$
\left\{\overline{\operatorname{grad}}\left(\operatorname{Mult}_{r, s_{r}}\right)\left(v_{r}\right) \mid s_{r} \in D_{r}, r \in R\right\}
$$


YURI G. ZARHIN (ZARKHIN)

is linearly dependent in $\mathbb{C}^{n}$. Here $v_{r}=\pi_{r}(v) \in \hat{Z}_{n, r}^{\prime}$. Clearly, $X_{\left\{D_{r} \mid r \in R\right\}}$ is a closed algebraic subvariety in $\hat{Z}_{n, R}$. Now let us consider the union $Y$ of all such $X_{\left\{D_{r} \mid r \in R\right\}}$ for all choices of $\left\{D_{r} \mid r \in R\right\}$. It is also clear that $Y$ is also a closed algebraic subvariety in $\hat{Z}_{n, R}$. Since $\pi$ is finite, the image $\pi(Y)$ is a closed algebraic subvariety in $Z\left(n, \ell ; r_{1}, r_{2}, \ldots, r_{\ell}\right)$. On the other hand, one may easily check that $Z^{0}\left(n, \ell ; r_{1}, r_{2}, \ldots, r_{\ell}\right)$ is the complement of $\pi(Y)$ in $Z\left(n, \ell ; r_{1}, r_{2}, \ldots, r_{\ell}\right)$. It follows that $Z^{0}\left(n, \ell ; r_{1}, r_{2}, \ldots, r_{\ell}\right)$ is Zariski-open in $Z\left(n, \ell ; r_{1}, r_{2}, \ldots, r_{\ell}\right)$. This ends the proof of Theorem 1.5.

Acknowledgements. I am grateful to Yulij S. Ilyashenko, Vladimir L. Popov and Victor S. Kulikov for stimulating discussions. My special thanks go to Tatiana Bandman, whose comments helped to improve the exposition.

Part of this work was done during my stay at Weizmann Institute of Science Department of Mathematics in May-June of 2012 and at Centre Interfacultaire Bernoulli (École Polytechnique Fédérale de Lausanne) in July-August of 2012: I am grateful to both of them for the hospitality.

\section{REFERENCES}

[1] G. Buzzard, S, Hruska, Yu. Ilyashenko, Kupka-Smale theorem for polynomial automorphisms of $\mathbb{C}^{2}$ and persistence of heteroclinic intersections. Invent. Math. 161 (2005), 45-89.

[2] Igors Gorbovickis, The homoclinical tangency is not typical for polynomial automorphisms of $\mathbb{C}^{2}$ that preserve the volume. Diploma work, Lomonosov Moscow State University, 2006.

[3] Victor S. Kulikov, Appendix to [11.

[4] I.G. Macdonald, Symmetric functions and Hall polynomials. First edition. Clarendon Press, Oxford, 1979.

[5] James Milne, Algebraic Geometry. http://www.jmilne.org/math/CourseNotes/ag.html.

[6] John Milnor (with an appendix by Milnor and Tan Lei), Geometry and dynamics of quadratic rational maps. Experimental Mathematics 2 (1993), 37-83.

[7] Elmer Rees, On a paper by Y.G. Zarhin. arXiv:1105.4156 [math.AG].

[8] J.-P. Serre, Lie algebras and Lie groups. Second edition. Springer Lecture Notes in Math. 1500 (1992).

[9] I.R. Shafarevich, Basic Algebraic Geometry, volume I. Second edition. Springer Verlag, 1994.

[10] Y. Yomdin, Singularities in Algebraic Data Acquisition. In: Real and Complex Singularities (M. Manoel, M. C. Romero Fuster, C. T. C. Wall, eds.), London Mathematical Society Lecture Notes 380, pp. 378-396. Cambridge University Press, 2010.

[11] Yu. G. Zarhin, Polynomials in one variable and ranks of certain tangent maps. Mat. Zametki 91:1 (2012), 539-550; Math. Notes 91:4 (2012), 508-516; arXiv:1010.0282 [math.AG].

Department of Mathematics, Pennsylvania State University, University Park, PA 16802, USA

Institute of Mathematical Problems of Biology, Russian Academy of Sciences, Pushchino, Moscow Region, Russia

E-mail address: zarhin@math.psu.edu 